\title{
Thanatology in a Liaison Psychiatry Setting
}

\author{
R. K. Shelley, Senior Registrar in Psychiatry, St Vincent's Hospital, Elm Park, Dublin; (formerly Senior Registrar in
} Psychiatry, St James's Hospital, Dublin)

There has been increasing interest in recent years in the care of the terminally ill. Much of what has been written has been focussed on medical management and centred in hospices. The psychological dimension often appears neglected. The objective of this article is to outline the evolution, practice and problems of a liaison psychiatry input to the care of patients with terminal illness treated in a general hospital.

A specific section of St James's Hospital, a large teaching hospital in Dublin, has been set aside for the sole treatment of haematological and oncological disorders. The patients are under the care of two consultant physicians, each a specialist in his respective field. A psychiatric consultation was requested only when a patient manifested an obvious psychological problem. After responding to a number of such requests, it became apparent that many emotionally distressed patients were in fact reacting appropriately. Furthermore, there were other patients whose reactions seemed less appropriate who were not being referred. Case management was complicated by the traditional reluctance of medical patients to engage in a therapeutic alliance with a psychiatrist, in particular someone they had never met before. To overcome this and other difficulties, it was thought necessary to involve the psychiatrist in patient management in a routine way. The hypothesis was that a psychiatric input would be more acceptable to both patients and staff if it was routinely built into the overall patient care.

Before making the greater service commitment that this would entail, it was thought essential to obtain prior agreement of all medical and nursing staff concerned; patients had readily agreed to its potential usefulness. This prior agreement was considered to be a prerequisite because, without it, further developments could prove impossible. A general consensus emerged and the necessary support was assured.

\section{The service}

Over a period of time the service evolved with two major inputs. Firstly, all patients newly admitted under the care of the two consultants with a likely diagnosis of a potentially terminal illness were routinely assessed by the psychiatrist. Secondly, a weekly meeting took place solely to discuss the psychological dimensions of the patients' illnesses. This meeting was attended by all medical and nursing staff, both senior and junior. A social worker and cleric, with an interest and experience in this work, were recruited. This broadened discussion to include important social and spiritual dimensions. The meeting also became a forum for the education of staff on psychological issues.

In a practical way, working with patients involved facilitating communication with their clinician, assessing their psychological reactions and both supporting and sanctioning appropriate expression of affect. It was ward policy to communicate openly with patients and not to hide relevant information from them unless there was a specific indication for so doing. Although this openness could prove initially upsetting, it proved acceptable in the long run, particularly as it allowed patients to have faith in optimistic medical reports and to perceive that reassurances, when given, had substance. The patients' emotional reaction to their illness was governed predominantly by personal factors, the defence mechanisms used, and the nature of their illness.

\section{The patients}

The patients with a normal premorbid personality and who were readily able to express feelings appeared to cope best. From the psychiatrist's point of view, the fact that one was dealing with predominantly normal personalities made the work less complicated than expected. The patient's past personal experience of death and loss often proved relevant. An unresolved painful loss sensitised them to their own potential death. If they had coped well with previous loss, this appeared to strengthen their own coping skills.

How patients formulated their illness, whether in religious or other terms, was also relevant. Most stated that they did not fear death, rather, they feared the process of dying. This was associated with apprehensions regarding pain, loss of body function, lack of dignity, as well as being a burden on their family. This sense of being a burden had many practical aspects, but it often refiected guilt feelings over the pain their death might cause to others.

\section{Psychological reactions}

Their initial emotional reactions were complicated by uncertainty. To know specifically what fate has in store allows adjustments, but not knowing whether or not one is in the $x \%$ which may respond well to chemotherapy created cognitive and emotional confusion; this precluded adjustment.

The patient's emotional reactions were principally depression, guilt, and anger. These are all understandable and the depression is clearly a mourning for one's potential loss of life. The loss is also the loss of health, loss of status that goes with ill health, and loss of future expectations for self and others in one's life. Guilt resulted from internalisation of some of these feelings. It focussed usually on a sense of guilt at deserting one's own family and could be expressed as a sense of guilt for not having sought medical assistance earlier. The anger manifested itself in a variety of ways, moving from cynicism, through being verbally abusive to refusal to co-operate with medical procedures. The anger could be projected onto fate, God, doctors for their failures and on to healthy people, especially those at a similar stage in life. These angry reactions often led into 
guilt, and the discussion of guilt and anger often proved one of the most fruitful areas during psychotherapy.

\section{Defence mechanisms}

The defence mechanism of denial was one used to a lesser or greater extent by all. In routine psychiatric practice, one tends perhaps to view denial as a pathological mechanism. When talking with the dying, one can see that it has a positive value. Indeed, it has been suggested by Greer ${ }^{1}$ that denial may improve the prognosis in terminal disease. However, in excess it becomes counter-productive, when it can either inhibit emotional resolution or even lead to refusal to comply with medical advice. The guilt and the anger mentioned above reflect the operation of internalisation and projective mechanisms. Many people also tended to devalue their own self worth. The more they were able to devalue themselves the less they perceived what their potential loss to others might be when they died. This enabled a reduction in their own sense of guilt.

This desire to protect others is also a defence mechanism and it might be termed 'altruism'. It manifested itself in other ways, such as when patients gradually distanced themselves from their families. They might isolate themselves for example during visiting hours, through silence or retreat behind a book, even to the point where they became quarrelsome and difficult with relatives. These actions were difficult to understand initially and had previously been thought to be the result of anger. However, on discussion with patients it became apparent that they felt that if they could push away those who were close to them, then when they died, their loss would be less to their families. They often recognised that what they were doing was painful to their relatives, but in an altruistic way they felt it was for the better-"I know the kids find me a bit irritable, but at least they won't miss me so much when I'm gone."

Other defences observed were identification and idealisation. There was a tendency to identify with other patients, particularly those with a similar disease. This could lead to identification through symptoms, and was often more likely with those patients whose illness appeared to be running a better course than theirs. Idealisation, particularly of the medical staff, was important. Patients needed to see their physician as omnipotent, which is understandable and sometimes useful. This, however, was something the staff needed to avoid feeding into as it could, for example, cause them to make therapeutic promises on which they could not reasonably expect to deliver.

\section{The illness}

The illness variables which influenced their psychological reactions included their premorbid perception of their diagnosed illness, such as associating cancer with stigma. The symbolic importance of the organ involved could be psychologically relevant, e.g. breast or ovarian carcinoma for a female. The patient's change in body image was important. This and other cognitive changes in the terminally ill appears a poorly understood and poorly researched area. The change from a body image of being healthy to being severely ill was generally difficult for many, especially for those for whom physical fitness was of great importance and who now felt betrayed by their own bodies.

Patient's adjustment was also complicated by their inability to observe their own pathology. A fractured limb with a plaster of Paris was something they could understand and adjust to, but a cancer was not. As one patient put it, "It's like shadow boxing," and "If only I could take a sledge hammer to it". In the absence of anything concrete their imagery and fantasy of their pathology could be bizarre. These changes in body image are often further exacerbated by the consequences of chemotherapy, such as when alopecia is induced, or when a female patient requires a Hickman atrial catheter.

\section{The stafi}

Many of the staff's attitudes to patients' emotional responses were dichotomised between seeing patients who are stoical as being 'good patients', and patients who are manifestly distressed as being 'psychiatrically ill'. Their discomfort in the presence of an emotionally distressed patient could manifest itself as a recommendation that the patient be placed on antidepressant medication. In the author's experience this is rarely if ever required in the terminally ill. Staff's own sense of guilt at not being able to help the terminally ill could similarly manifest itself as pressure on consultant physicians "to do something", even when further aggressive treatment was clearly inappropriate. The patient-staff relationship was often characterised by transference/countertransference issues. Patients often saw their physician as the idealised good father. For patients who are chronic attenders the transference was often for the institution rather than individuals. Staff's own reactions to patients seemed at times to have countertransference elements. However, in general, staff felt threatened by any attempt to discuss transference/countertransference with them.

\section{The psychiatrist}

Anyone involved in psychological aspects of thanatology needs to be aware of their own defence mechanisms. Repression and denial may manifest as avoidance or forgetting, or rationalisation as 'finding something more important to do', or even identification with patients' symptoms. As with all psychotherapeutic interactions, transference and countertransference operate. It is therefore advised that appropriate and regular supervision be available.

Finally, work in this area offers challenges at many different levels. However, with careful planning and adequate support, the patients themselves can make it rewarding.

REFERENCE

${ }^{1}$ Greer, S. et al. (1979) Psychological response to breast cancer: effect on outcome. Lancet, 2, 785-787. 